\title{
əParameter Optimization for Real-World ENSO Forecast in an Intermediate Coupled Model
}

\author{
YUCHU ZHAO \\ Department of Atmospheric and Oceanic Sciences, Peking University, Beijing, China \\ ZHENGYU LIU \\ Atmospheric Science Program, Department of Geography, The Ohio State University, Columbus, Ohio, and \\ Department of Atmospheric and Oceanic Sciences, Peking University, Beijing, China \\ FEI ZHENG \\ Institute of Atmospheric Physics, Chinese Academy of Sciences, Beijing, China \\ YISHUAI JIN \\ Department of Atmospheric and Oceanic Sciences, Peking University, Beijing, China \\ (Manuscript received 4 June 2018, in final form 10 January 2019)

\begin{abstract}
We performed parameter estimation in the Zebiak-Cane model for the real-world scenario using the approach of ensemble Kalman filter (EnKF) data assimilation and the observational data of sea surface temperature and wind stress analyses. With real-world data assimilation in the coupled model, our study shows that model parameters converge toward stable values. Furthermore, the new parameters improve the real-world which controls the strength of anomalous upwelling advection term in the SST equation. The improved prediction skill is found to be contributed mainly by the improvement in the model dynamics, and second by the improvement in the initial field. Finally, geographic-dependent parameter optimization further improves the prediction skill across all the regions. Our study suggests that parameter optimization using ensemble data assimilation may provide an effective strategy to improve climate models and their real-world climate predictions in the future.
\end{abstract} \\ ENSO prediction skill, with the skill improved most by the parameter of the highest climate sensitivity (gam2),
}

\section{Introduction}

Recent studies have shown that data assimilation may be used for improving weather and climate models by estimating model parameters with the observational data. Most of these studies, however, have been performed in the perfect model scenario (Aksoy et al. 2006a; Tong and Xue 2008a,b; Wu et al. 2012; Zhang et al. 2012). These studies show that model parameters can indeed converge toward the truth values, even in coupled general circulation models (CGCM; Liu et al.

¿ Denotes content that is immediately available upon publication as open access.

Corresponding authors: Yuchu Zhao, 1401110225@pku.edu.cn; Zhengyu Liu,liu.7022@osu.edu
2014; Li et al. 2018). With improved parameters, model bias can be reduced (Tong and Xue 2008a,b) and the prediction skill can be improved (Wu et al. 2012; Zhang et al. 2012).

Since our ultimate objective of making weather and climate models is for the study of the real world, it is essential to explore parameter estimation with observational data. So far, however, there have been few studies on the parameter estimation for the real world. In general, parameter estimation with observational data poses further challenges. First, models inevitably have deficiencies and biases compared with the real world (Zheng and Zhu 2016). Second, for the real-world scenario, it is not even clear if most of the model parameters have the "truth" values. Third, for some parameters that do have optimal, it is not clear if the optimal values would be state-dependent and therefore are no longer 
TABLE 1. The parameters for estimation.

\begin{tabular}{|c|c|c|}
\hline Parameter & Physical meaning & Default value \\
\hline gam1 & $\begin{array}{l}\text { Strength of mean upwelling } \\
\text { advection term in SST } \\
\text { equation }\end{array}$ & 0.75 \\
\hline $\operatorname{gam} 2$ & $\begin{array}{l}\text { Strength of anomalous upwelling } \\
\text { advection term in SST } \\
\text { equation }\end{array}$ & 0.75 \\
\hline tda1 & $\begin{array}{l}\text { Amplitude of subsurface } \\
\text { temperature anomaly for } \\
\text { positive } h \text { perturbations }\end{array}$ & 28 \\
\hline tda2 & $\begin{array}{l}\text { Amplitude of subsurface } \\
\text { temperature anomaly for } \\
\text { negative } h \text { perturbations }\end{array}$ & -40 \\
\hline $\operatorname{tdb} 1$ & $\begin{array}{l}\text { Affect the nonlinearity of } \\
\text { subsurface temperature } \\
\text { anomaly for }+h \text { perturbations }\end{array}$ & 1.25 \\
\hline $\operatorname{tdb} 2$ & $\begin{array}{l}\text { Affect the nonlinearity of } \\
\text { subsurface temperature } \\
\text { anomaly for }-h \text { perturbations }\end{array}$ & 3 \\
\hline
\end{tabular}

temporally constant (Hansen and Penland 2007). Indeed, for the real-world scenario, since the true value of a parameter is unknown, it becomes sometimes unclear how to judge if a parameter achieves the "optimal" value. An optimal parameter can be judged on its effect in improving the model simulation and model biases, it can also be judged on its effect on the improvement of the real-world prediction. In the few examples available, Menemenlis et al. (2005) and Toyoda et al. (2015) performed parameter estimation in OGCMs with Green's function method and suggested that the optimized parameters can improve the model simulation. $\mathrm{Hu}$ et al. (2010) performed simultaneous state and parameter estimation in a weather model and suggested that the optimized parameters are able to improve the model forecast skill for real-world weather events. In a simplified intermediate coupled model (ICM), Kondrashov et al. (2008) performed parameter estimation with observational data and suggested that the optimized parameters improve the model simulation such that the simulation tracks the observations better.

Here, different form previous studies, we study parameter estimation with real-world observations in an ICM with the focus on the model prediction skill. We will use the Zebiak-Cane model (Zebiak and Cane 1987) in its latest version LDEO5 (Chen et al. 2004). Our study shows that, for real-world observations, key model parameters converge to new optimal values and the optimized parameters can improve the ENSO forecast skill significantly. The paper is organized as follows. Section 2 briefly describes the model and the method of parameter estimation. Section 3 shows the estimation results and the prediction skill in the ZC model. Section 4 discusses

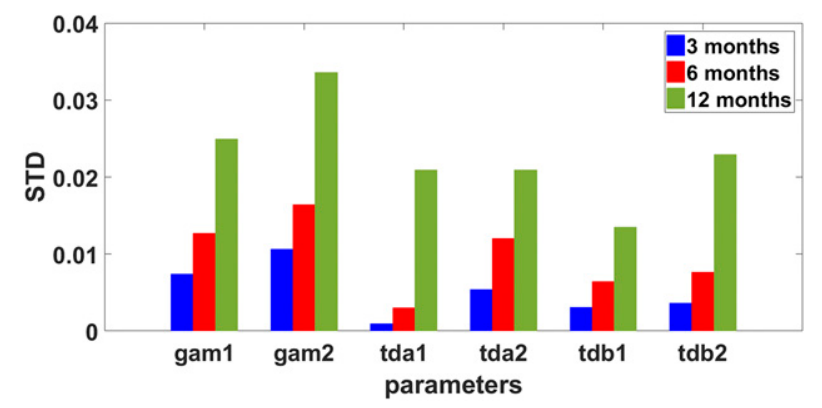

FIG. 1. Model sensitivity for six parameters. For each parameter, the sensitivity is obtained by perturbing the parameter by $10 \%$ of its default value at the initial time in a 100 -member ensemble run and force the models for 1 year; then the 3-month (blue), 6-month (red), and 12-month (green) mean standard deviation of SST is used to represent the sensitivities.

further issues related to the postprocessing process of the forecast and the effect of estimating spatially varying parameters. A summary is given in section 5 .

\section{Model and method}

\section{a. Model description}

We use the well-known intermediate coupled oceanatmosphere model Zebiak-Cane (ZC) model (Zebiak and Cane 1987) in its latest version, Lamont-Doherty Earth Observatory, version 5 (LDEO5; Chen et al. 2004). The model has been shown to be able to predict all prominent El Niño events in the 1857-2003 period successfully, with lead times up to 2 years (Chen et al. 2004). The atmospheric model is built on the steady-state, linear shallow water equations, with the atmospheric circulation driven by a heating anomaly associated with moisture convergence and SST anomaly (SSTa). The ocean model is built on the reduced gravity model, and the ocean currents are generated initially by the observed monthly wind field, and then driven by the wind stress anomaly of the atmosphere model. There is also an equation for the SSTa (appendix A), which depends on the heat flux exchange between the surface and subsurface waters as well as the surface heat flux and advection. The model ocean domain is the tropical Pacific Ocean region $\left(28.75^{\circ} \mathrm{S}-28.75^{\circ} \mathrm{N}, 124^{\circ} \mathrm{E}-80^{\circ} \mathrm{W}\right)$. The atmosphere and SSTa have a resolution of $5.625^{\circ}$ (longitude) $\times 2^{\circ}$ (latitude), while the ocean dynamics have a resolution of $2^{\circ}$ (longitude) $\times 0.5^{\circ}$ (latitude).

Compared to the original ZC model, the LDEO5 model has an additional bias-correction module, which significantly improves the forecast skill for the real world. The bias-correction method is based on the regression of model errors with model state variables in a reduced space of empirical orthogonal functions and 

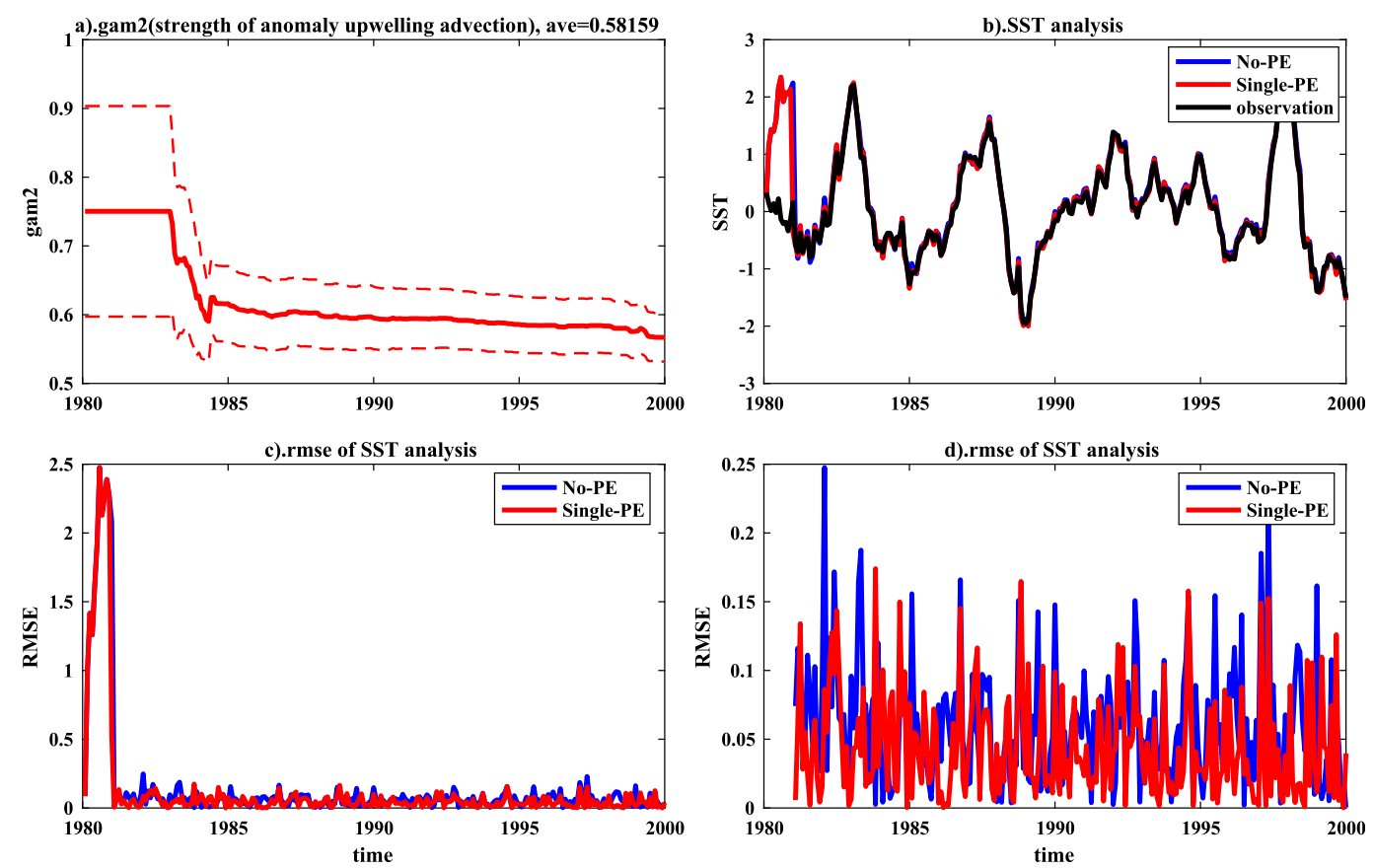

FIG. 2. (a) Parameter estimation of gam2. The ensemble size is 100 . The observations are the monthly SST anomaly. The red solid line is the temporal evolution of the ensemble mean of gam2, and the red dashed lines are the one standard deviation of its ensemble spread. (b) Niño-3.4 SSTa and (c) RMSE of Niño-3.4 SSTa analysis in No-PE and Single-PE experiments. (d) As in (c), but for the period of 1981-2000 (remove the first year without data assimilation).

is applied at every time step of the forecast (Chen et al. 2000).

\section{b. Method and observation data}

We use the ensemble Kalman filter (EnKF) for data assimilation (Evensen 1994; Zheng et al. 2006). The parameters are estimated by augmenting the state variables with model parameters in a simultaneous estimation of both the state and model parameters (Anderson 2001). Similar results are also obtained when we use the ensemble adjustment Kalman filter (EAKF) method (Anderson 2001; not shown). The details of the EnKF scheme and parameter estimation scheme are in appendix B. Briefly, here, for each experiment, parameter estimation is carried out after a spinup period in which only the state variables are updated until the model state ensembles are constrained by observation sufficiently (Zhang et al. 2012). We use an ensemble size of 100 and perform the data assimilation and parameter estimation at the last time step of each month. We have tested assimilation periods from 1 to 3 months and the result is insensitive to the length of the assimilation. To avoid the accumulation of sampling error during the update process, we use a spatial updating method (Aksoy et al. 2006a) that transforms a globally uniform parameter to a two-dimensional field and update it grid-by-grid using localization. A covariance localization (Gaspari and Cohn 1999) is applied to both the state and model parameters within the area of 3 longitude grids and 7 latitude grids. To avoid the loss of ensemble spread, a conditional covariance inflation (CCI) technique (Aksoy et al. 2006b) is also applied in the parameter estimation such that the ensemble spread is maintained above a prescribed threshold. The observational data are the monthly analyses of HadISST and Florida State University (FSU) wind stress (Goldenberg and O'Brien 1981) both in the period of 1980-2000. All observations are first interpolated to the model grid point before assimilation. The observed data are regarded as the "truth," and the "observations" used in data assimilation and parameter estimation systems are constructed by the addition of a Gaussian white noise onto the truth (Lu et al. 2017). In our study, model SSTa and wind stress anomaly are corrected by all observations within the localization distance of the cross covariance (Gaspari and Cohn 1999). An additive inflation technique is also used for model state (Hamill and Whitaker 2005). Model parameters are estimated with the observed SST anomaly only. The errors of the SSTa and the wind stress anomaly in the observation are assumed independent and have the scales of $0.1 \mathrm{~K}$ (Karspeck and Anderson 2007) and $0.1 \mathrm{~m} \mathrm{~s}^{-1}$, respectively. In all of our study shown 

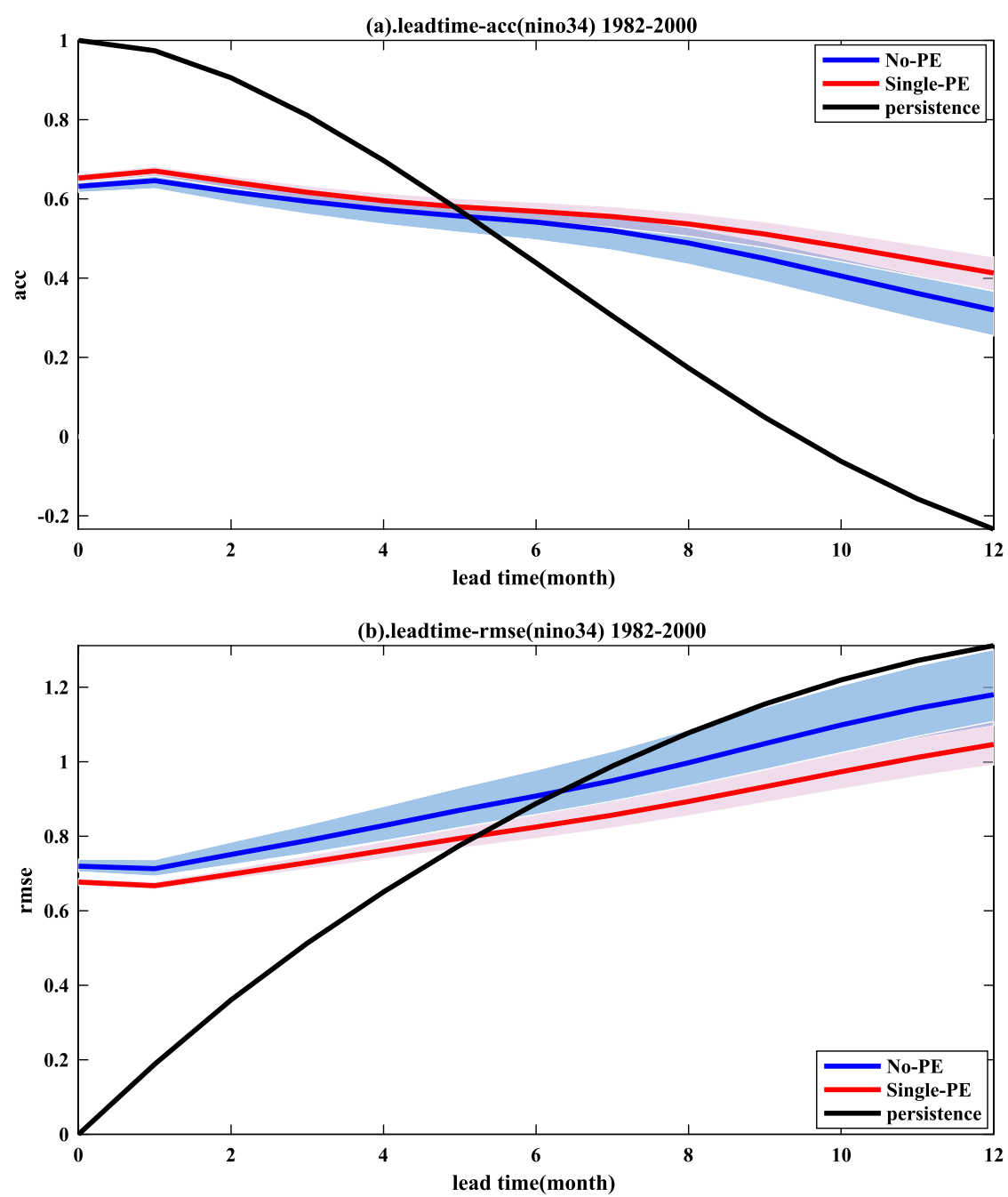

FIG. 3. ZC model prediction skill of the Niño-3.4 index in the period of 1982-2000 with the lead time up to 12 months. The blue line is the skill of model forecast ensemble mean with default parameter value. The red line is the skill of model forecast ensemble mean after single-parameter estimation for gam2. The shading is the one standard deviation of spread. The black line is the skill of persistence prediction. (a) ACC and (b) RMSE.

below, parameter estimation is made using the observation of 1980-2000 and the climate prediction is made for the SSTa in 1982-2000. We have tested other periods of the observations and our results remain largely unchanged.

\section{Parameter estimation and prediction skill in $\mathrm{ZC}$ model}

\section{a. Parameters in ZC model}

We first study the parameter estimation in the original ZC model such that there is no bias correction in the forecast. Six parameters in the SST equation are updated in our study (Table 1, appendix A). These parameters control the upwelling and subsurface temperature in the ocean and are therefore important for the simulation of SSTa and atmospheric circulation. Different parameters have different impacts on the model simulation, suggesting that different parameters have different model sensitivities. In our study, the model sensitivity is evaluated by perturbing a parameter with $10 \%$ of its default value and then calculate the 1 year mean standard deviation of SSTa after the perturbation. Our sensitivity experiments show that the final results are not sensitive to the initial perturbation magnitude. In addition, all the parameter estimation experiments below have been first carried out in the perfect model scenario. In all the perfect model experiments, all the parameters converge 

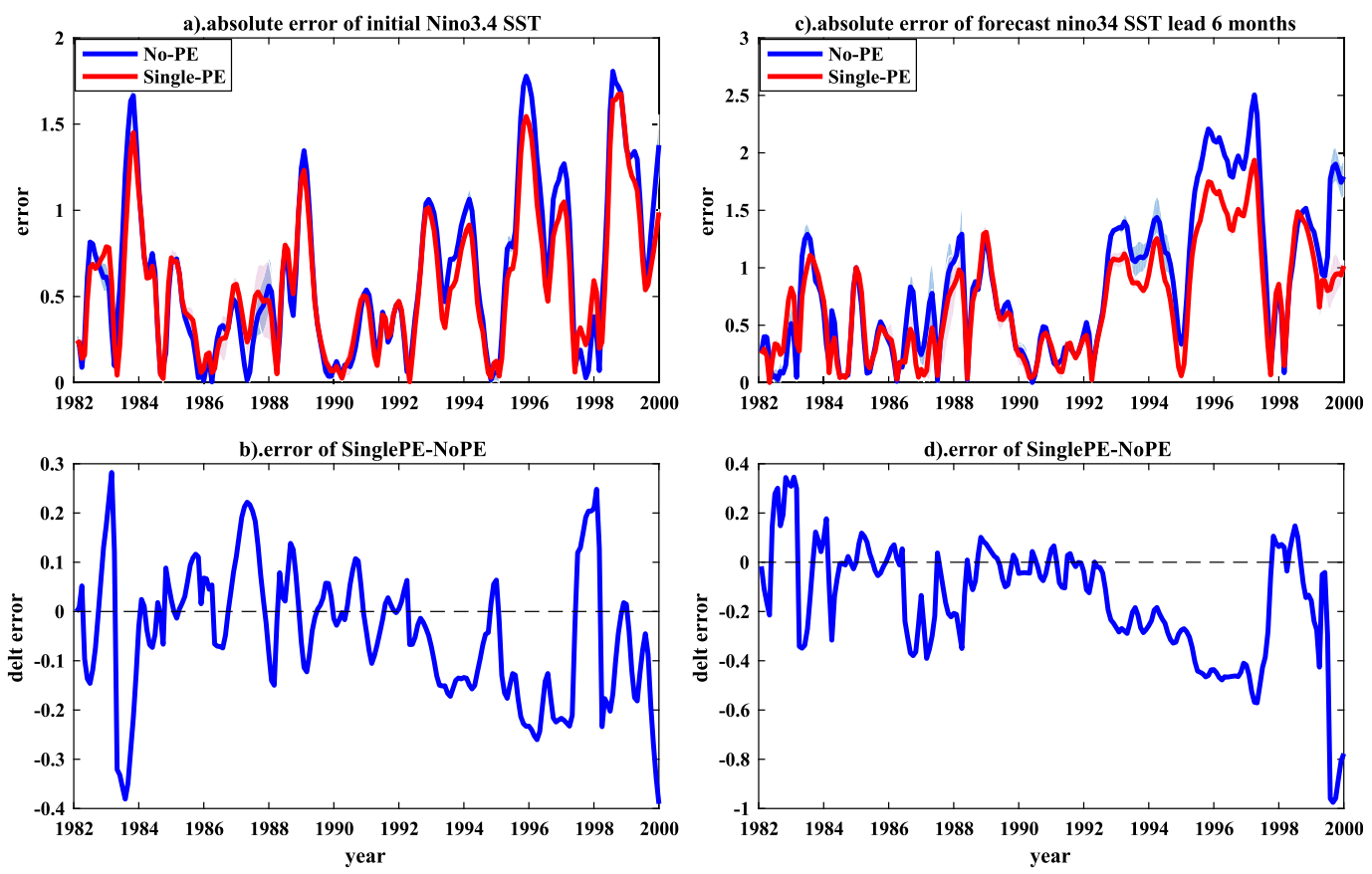

FIG. 4. Prediction error in the Niño-3.4 region for the period of 1982-2000 for the model with the default parameter gam2 (blue) and optimized parameter (red) (a) at the initial time and (b) the difference between Single-PE and No-PE, (c) at lead time of 6 months, and (d) the difference between Single-PE and No-PE. The solid is the ensemble mean and the shading is the one standard deviation of the spread.

toward the truth values. The challenge is therefore if they converge for real-world observation.

\section{b. Single-parameter estimation}

Our model sensitivity experiments for the six parameters above show that the most sensitive parameter is gam2 (Fig. 1), which controls the strength of anomalous upwelling advection term in the SST equation (appendix A) and in turn upwelling feedback in the coupled model. We therefore first perform singleparameter estimation (PE) for gam2 (Fig. 2). The parameter is updated every month for 20 years. It is seen that the parameter converges to a constant after 2 years. The optimized parameter value is calculated as the average of the last 10 years, which is 0.582 in this case. It should be pointed out that the convergence to a constant is not an obvious result for realworld parameter estimation. As pointed out by Hansen and Penland (2007), for the case of real-world assimilation, a model parameter may not even have a truth value, or it may not converge to a constant because the model parameter may depend heavily on state variables. Furthermore, Figs. $2 \mathrm{c}$ and $2 \mathrm{~d}$ show a reduction of the SST error in the analysis of Single-PE (red) compared with No-PE (blue).

Here, the optimized parameter will be evaluated in terms of the ensemble forecast skill. Specifically, we will compare the climate predictions between the model with the default parameter value (i.e., the model with no parameter estimation, hereafter No-PE experiment) and the model with the optimized parameter value (Single-PE experiment). Without further specification, we will use the Niño-3.4 SST anomaly as the index for comparison. All conclusions remain qualitatively unchanged if the forecasted SSTa averaged over the entire model region is used (not shown). The forecast skill is evaluated using both the anomaly correlation coefficient (ACC) and the root-mean-square error (RMSE) of the forecasted ensemble mean against the observation of 1980-2000. Figure 3 shows the prediction skills of lead times up to 12 months for Niño-3.4 SST anomaly in for the ensemble mean forecast (solid line) and the ensemble spread (one standard deviation in shading). It is seen that the model with the optimized parameter improves the forecast skill over the model with the default parameter, in both the ACC and RMSE (Figs. 3a,b, red vs blue solid lines). Indeed, the improvement, which is small in the initial month, becomes significant afterward 2 months of forecast lead time. This occurs because the initial forecast depends mostly on the initial field, while the influence of the parameter becomes dominant at longer lead times (Zhu and Navon 1999). Furthermore, the accuracy of forecast is also improved with the optimized parameter, which can be seen in the reduced 
(a).auto-correlation of NINO3.4 index (obs)

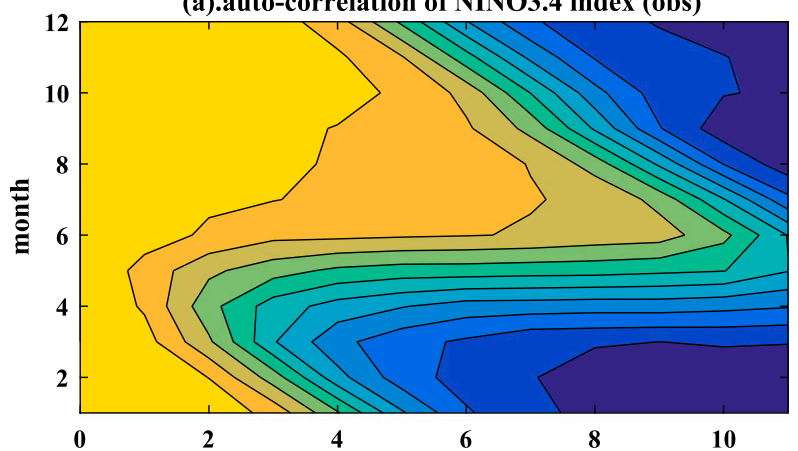

(b).auto-correlation of NINO3.4 index (No-PE)

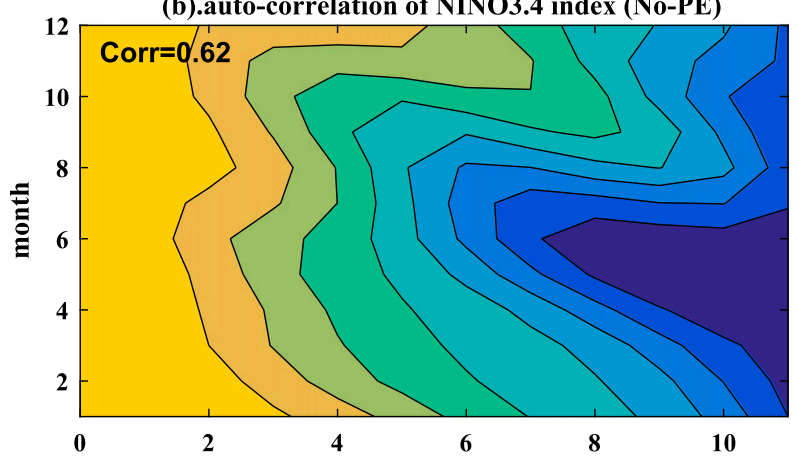

(c).auto-correlation of NINO3.4 index (Single-PE)

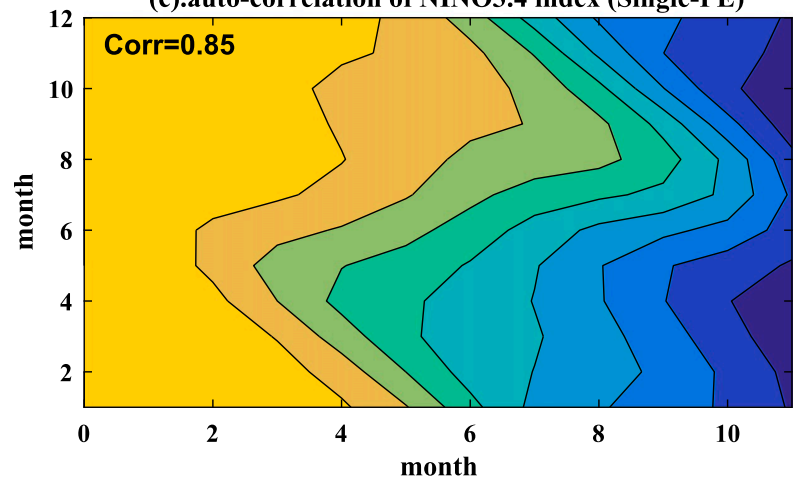

FIG. 5. Seasonal autocorrelation function from lag month 1 to 11 for the Niño-3.4 index in the (a) observation, (b) the model with the default parameter, and (c) the model with the optimized parameter gam2. The pattern correlation with observation is 0.85 (Single-PE) and 0.62 (No-PE).

forecast skill uncertainty (shading in Fig. 3). This result demonstrates that the single-parameter estimation of the parameter gam 2 can improve the prediction of this model for the real-world scenario. As a reference, the forecasts in both models beat the persistence forecast of the SST anomaly after $\sim 5$ months (Figs. 3a,b, black solid). The poor initial forecast of the model compared with the persistence is due to the error in the initial SSTa field. The model ocean initial state is obtained using the observed wind stress forcing in the coupled model (Chen et al. 2004) and therefore the initial SSTa differs from the observation. The persistence forecast, which is performed as $\operatorname{SSTa}(\mathrm{t})=\operatorname{SSTa}(0)$,

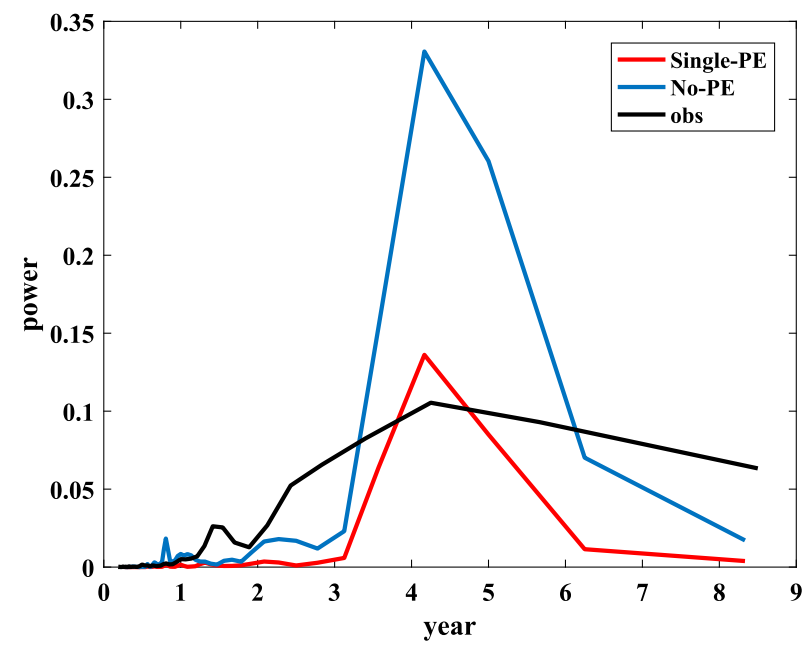

FIG. 6. Power spectrum density (PSD) of Niño-3.4 index. This PSD is obtained from the autocorrelation function. More specifically, we first calculate the autocorrelation function of the SST time series, and then apply the discrete Fourier transformation to derive the power spectrum density. The black line is the observation, the blue line is the model with the default parameter value, and the red line is the model with the optimized parameter value.

however, uses the observation SSTa itself as the forecast and is therefore "perfect" at the initial time $(\mathrm{ACC}=1, \mathrm{RMSE}=0)$ because the SSTa observation itself is used here for the evaluation of the forecast skill.

The forecast skill is increased because the model of optimized parameter improves both the initial field and the model itself. The ensemble initial state is obtained by forcing the coupled model with perturbed observational wind stress from 1970 to 2000 (Chen et al. 2004) and in this process, parameters can influence the obtained initial field. The model itself represents the model dynamics and the characteristics of model simulation, such as persistence, amplitude, and autocorrelation. The improvement of the initial field can be seen in the forecast skill at lead time 0 together with the error of the Niño-3.4 SST anomaly (Fig. 4). In our model prediction here, the ensemble initial field is obtained by the model with the default parameter for No-PE (Chen et al. 2004), and by the model with the optimized parameter for the Single-PE. A comparison of the errors of the two initial fields shows a clear reduction in the latter model, mainly in the period of 1992-2000 (Figs. 4a,b). Further error reduction can be seen in the forecast error at larger lead times, for example, at month 6 (Figs. 4c,d), which shows an error reduction in the whole period and is large in the period of 1992-2000.

The improvement on the model can be seen in the seasonal persistence or the seasonal autocorrelation 
(a).leadtime-acc(nino34) 1982-2000

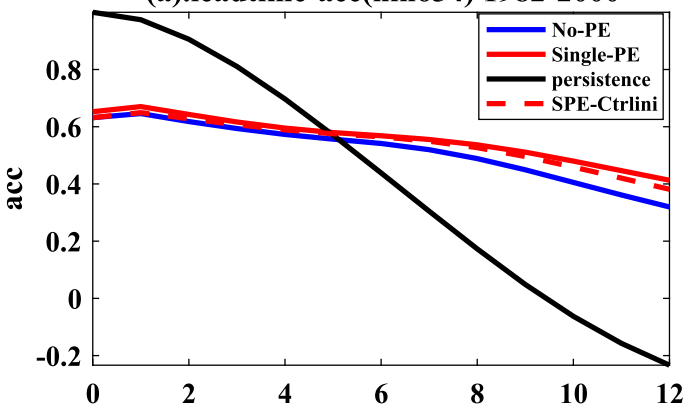

(c).leadtime-rmse(nino34) 1982-2000

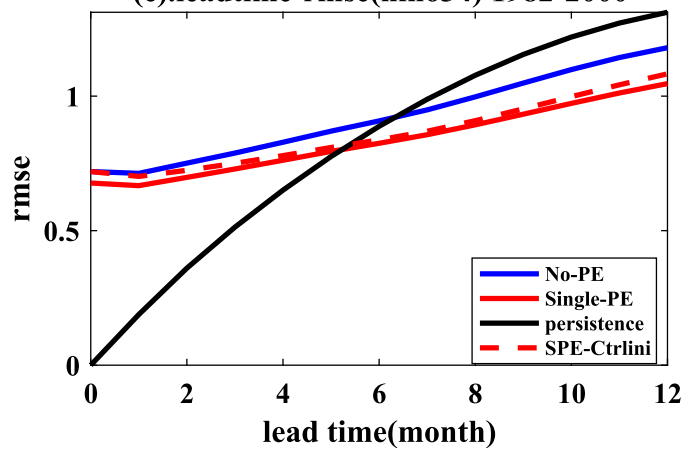

(b).leadtime-acc(nino34) 1982-2000

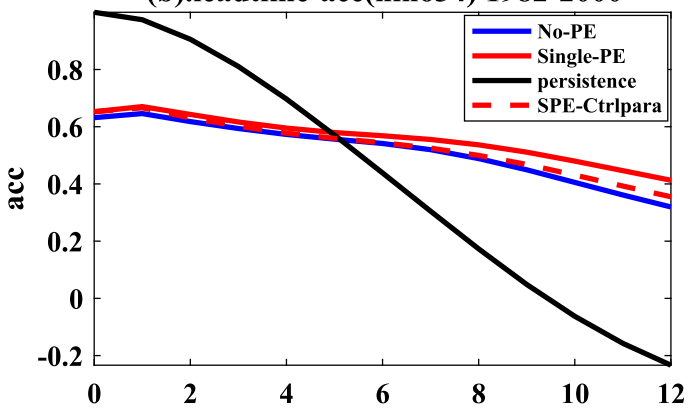

(d).leadtime-rmse(nino34) 1982-2000

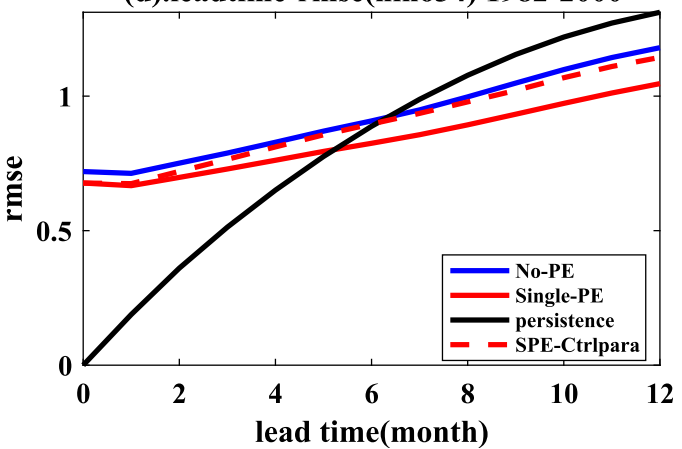

FIG. 7. As in Fig. 3, but only for the ensemble means. Additionally, we also plot two sensitivity experiments: (a),(c) SPE-Ctrlini (red dash), in which the forecast is made using the optimized parameter, but the No-PE initial field; (b),(d) SPE-Ctrlpara (red dash), in which the forecast is made using the default parameter but the initial field from the Single-PE.

function. Figure 5 shows the seasonal persistence map for the observation (Fig. 5a), No-PE (Fig. 5b). and SinglePE (Fig. 5c). It is seen that the persistence of No-PE fails to capture some major features of the observation, such as the sharp spring persistence barrier (Torrence and Webster 1998). This deficiency is improved with the optimized parameter. Indeed, the spatial correlation of the persistence map with the observation is improved substantially from 0.62 in NoPE to 0.85 in Single-PE. In addition, the optimized gam2 also seems to improve the intensity of the simulated ENSO somewhat. As seen in the power spectrum (Fig. 6), ENSO magnitude in No-PE is too large compared with the observation, and this excessive magnitude is reduced by the optimized parameter in Single-PE because of a smaller gam2, which reduces the upwelling response to wind stress, and in turn the upwelling positive feedback

To investigate the effects of the improvement of the initial field and the model itself on forecast skill separately, we performed two more experiments. In one experiment, the forecast initial field is the same as in No-PE but the forecast is made with the model of the optimized parameter (as in Single-PE; named SPE-Ctrlini; Figs. 7a, c, dashed line). In the other experiment, the forecast initial field is the same as in Single-PE but the forecast is made with the model of the default parameter (as in No-PE; named SPE-Ctrlpara; Figs. 7b,d, dashed line). The forecast skill of SPE-Ctrlini starts the same as that in No-PE, as expected, and then becomes close to Single-PE after 3 months (Figs. 7a,c). To the contrary, the forecast skill of SPE-Ctrlpara starts the same as that in Single-PE and then becomes close to No-PE after 3 months. This indicates that the impact of the optimized parameter on model dynamics contributes more to the model forecast skill, compared to that on initial field.

We have also performed single-parameter estimations on the other five parameters individually. The results are qualitatively similar. That is, in each case, the parameter converges to a new value. The new parameter improves the forecast skill in both ACC and RMSE. Quantitatively, however, the improvement of the forecast is smaller than that for gam2, consistent with their model sensitivities in Fig. 1.

It will be interesting to explore the physical mechanism that enables each optimized parameter to improve the model and the forecast. This understanding would involve extensive sensitivity experiments and analysis and therefore will be left for future studies. Here, we note that some features of the improvement are reasonable, physically. For example, the optimized gam 2 is smaller than the default by $\sim 20 \%$. A smaller 

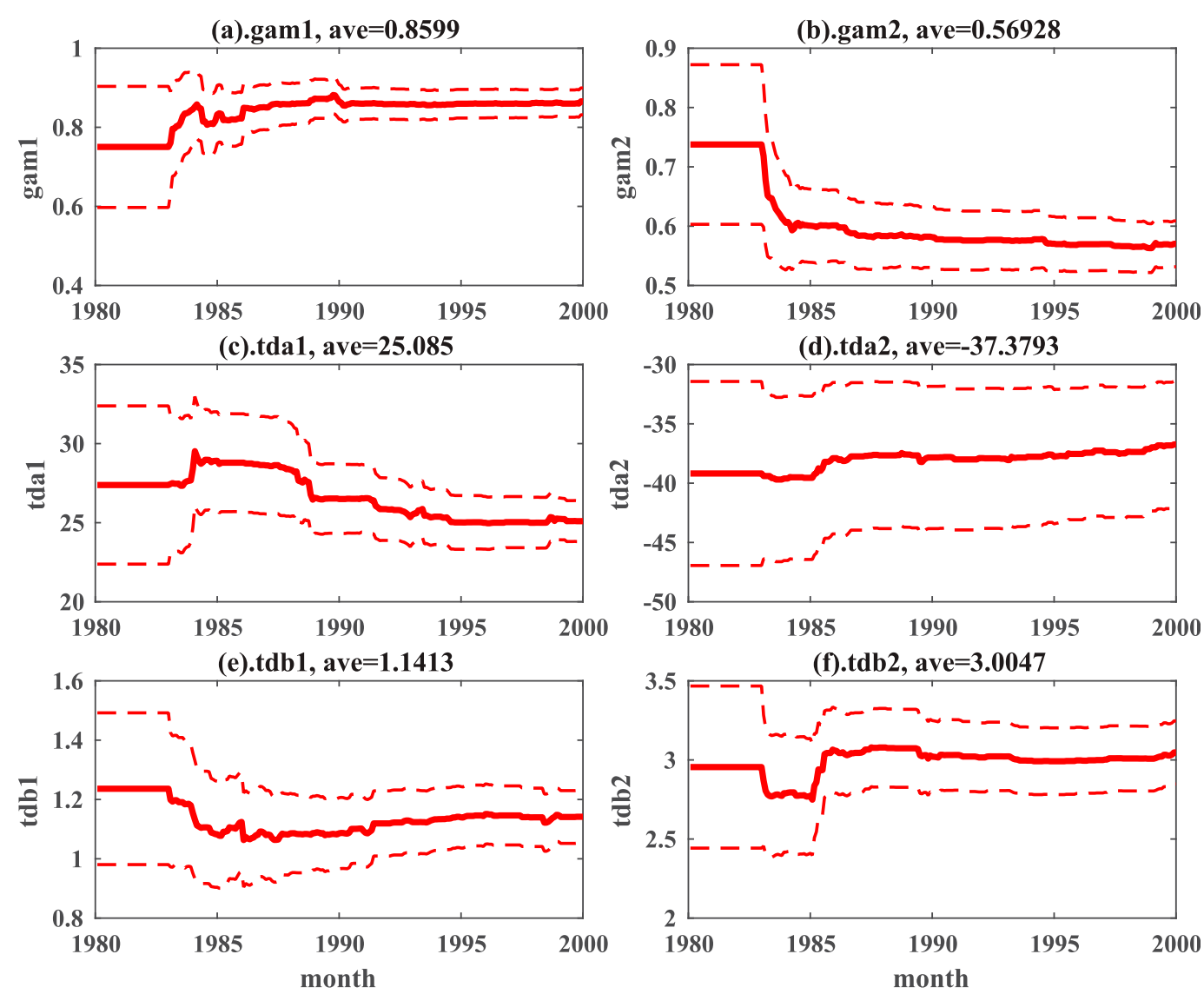

FIG. 8. Multiple-parameter estimation of the six parameters. The ensemble size is 100 . The observations are the monthly SST anomaly. The red solid line is the temporal evolution of the ensemble mean of each parameter, and the red dashed lines are the one standard deviation of its ensemble spread: (a) gam1, (b) gam2, (c) tda1, (d) tda2, (e) tdb1, and (f) tdb2.

gam2 leads to a weaker Ekman upwelling feedback, a weaker coupled feedback and, eventually, a weaker ENSO. This is consistent with the reduction of the ENSO amplitude. The improved seasonal cycle of the feedback may also contribute to the improvement of the spring persistence barrier here (e.g., Torrence and Webster 1998; Liu et al. 2019).

\section{c. Multiple-parameter estimation}

We now extend the parameter estimation from a single parameter to multiple parameters. We will estimate all the six parameters in Table 1 simultaneously. Figure 8 shows that all the parameters converge to constants in several years. The convergence value for each parameter is similar to that in the singleparameter estimation experiment. For example, the gam2 parameter starts from the default value of $\sim 0.75$ and converges toward $\sim 0.58$, almost the same as that in the single-parameter estimation (Fig. 2). This suggests that the parameter convergence is robust and rather independent of each other.
The model after multiparameter optimization (Multi-PE) shows some further improvements of the forecast skill over the best single-parameter case of gam2. This can be seen in the prediction of Niño-3.4 SST anomaly as in the single-parameter case (Fig. 9). The forecast skill of Multi-PE is improved over the gam2 optimization forecast both in ACC and RMSE (cyan line). The improvement is consistent with singleparameter estimation experiments for other parameters (not shown).

Finally, similar to the Single-PE case, the improvement of the forecast skill is contributed by both the initial field and the model itself. As in the Single-PE case, two experiments MPE-Ctrlini and MPE-Ctrlpara are also performed to investigate the separate impacts of the initial field and model itself on the forecast skill (Fig. 10). Again, similar to the case of single-parameter estimation, the MPE-Ctrlini starts with the same skill as No-PE, but then becomes close to Multi-PE (Figs. 10a,c); the MPE-Ctrlpara starts with the same skill as Multi-PE, but then becomes close to No-PE; therefore, 
(a).leadtime-rmse(nino34) 1982-2000

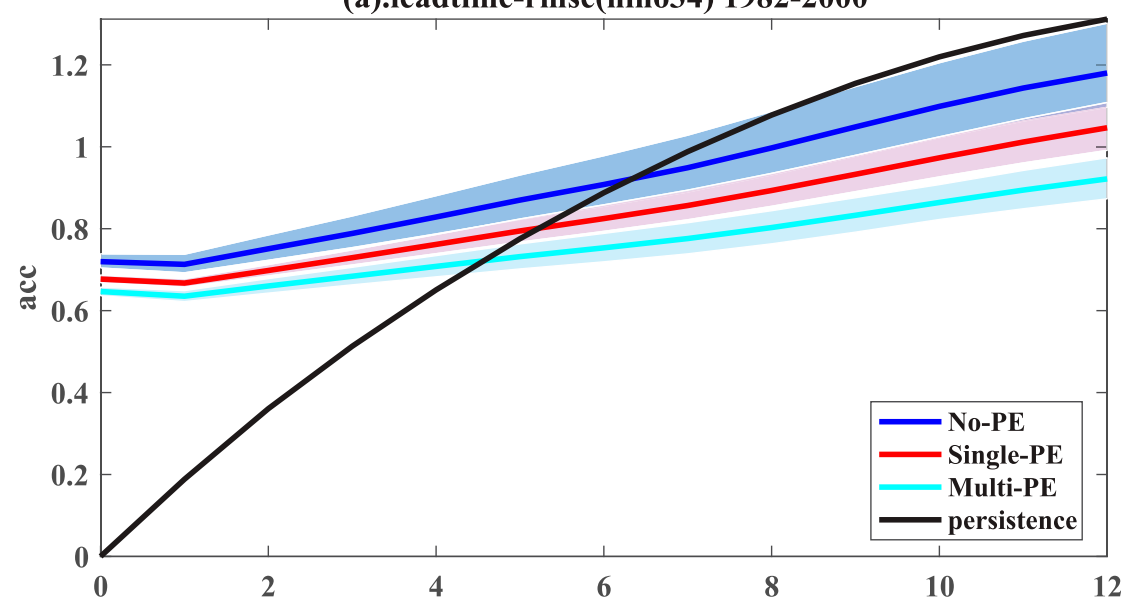

(b).leadtime-acc(nino34) 1982-2000

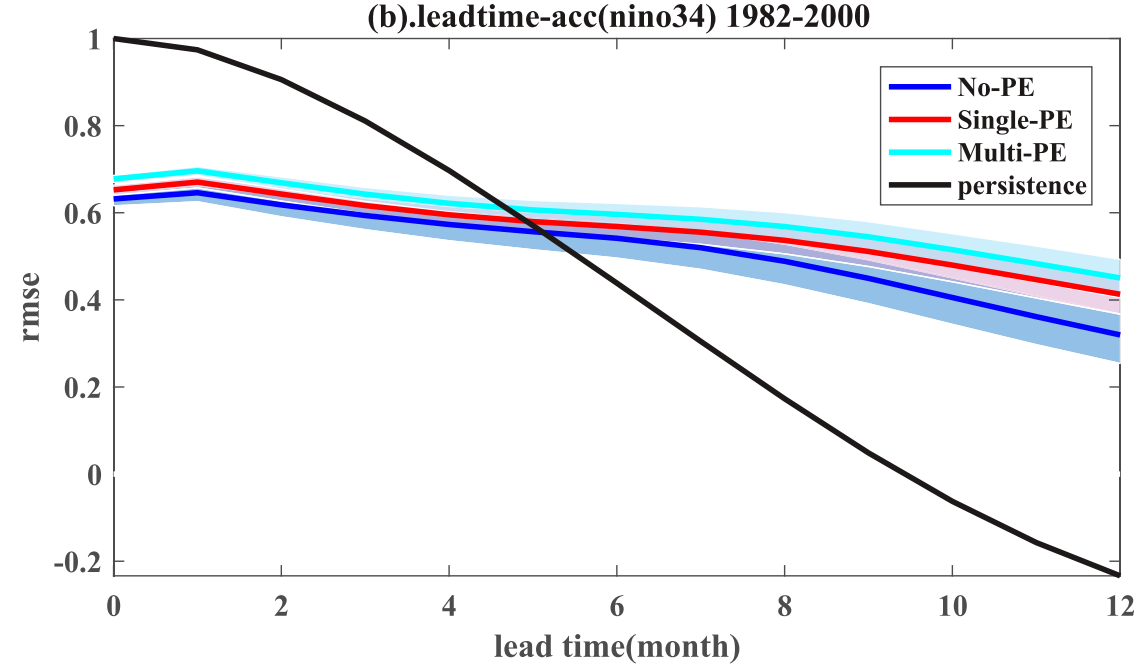

FIG. 9. Model prediction skill of Niño-3.4 index in 1982-2000 with different lead time in the multiple-parameter estimation case. The blue line is the skill of model prediction with the default parameter values. The red line is the skill of model prediction after single-parameter estimation. The cyan line is the skill of model prediction after multiple parameter estimation. The shading is the one standard deviation of spread. (a) ACC and (b) RMSE.

the impact of optimized parameters on model itself is more important than that on initial field for the forecast.

\section{Parameter estimation and prediction skill in LDEO5}

\section{a. Single- and multiple-parameter estimation}

The original ZC model discussed in section 3 has a low prediction skill. For example, after 6 months, the forecast ACC is reduced to below $\sim 0.5$ and the RMSE is increased to $\sim 1^{\circ} \mathrm{C}$. This climate forecast skill in the $\mathrm{ZC}$ model is improved significantly in the LDEO5, which consists of the ZC model and an additional biascorrection module. The latter improves the forecast skill significantly with an interactive statistical correction at every time step. This correction is based on the regression between the leading empirical orthogonal functions (EOFs) of the model errors and the leading multivariate EOFs of the model states (Chen et al. 2004). As such, for example, at month 6, the forecast ACC is increased to $\sim 0.75$ (Fig. 11a, blue) and the RMSE is reduced about a half to $\sim 0.66^{\circ} \mathrm{C}$ (Fig. $11 \mathrm{~b}$, blue).

To investigate the parameter estimation scheme in the model with the bias-correction module, we perform two types of experiments. In the first type of experiments, we perform the parameter estimation in the model without bias correction as in section 3 and evaluate the optimized parameters by the forecast with the bias-correction module. The results show that both the single and 
(a).leadtime-acc(nino34) 1982-2000

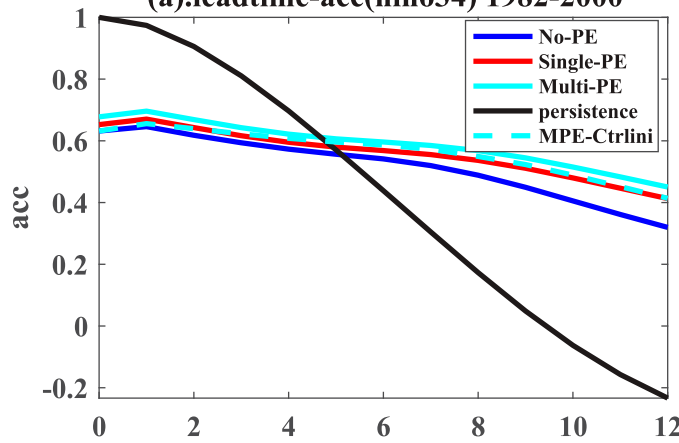

(c).leadtime-rmse(nino34) 1982-2000

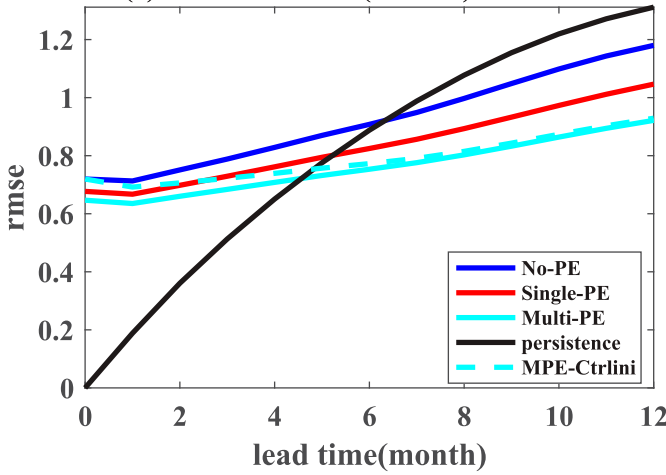

(b).leadtime-acc(nino34) 1982-2000

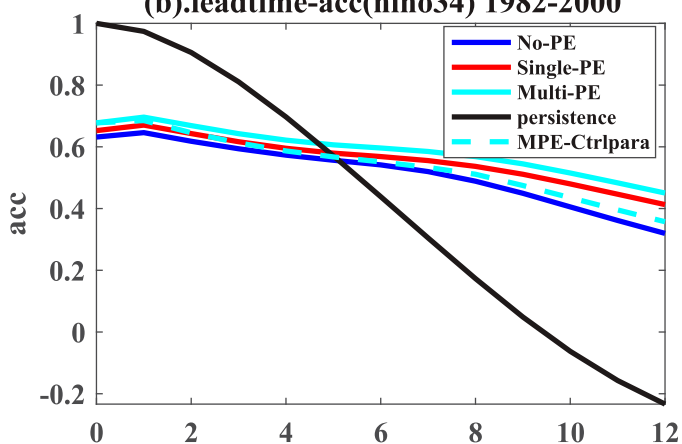

(d).leadtime-rmse(nino34) 1982-2000

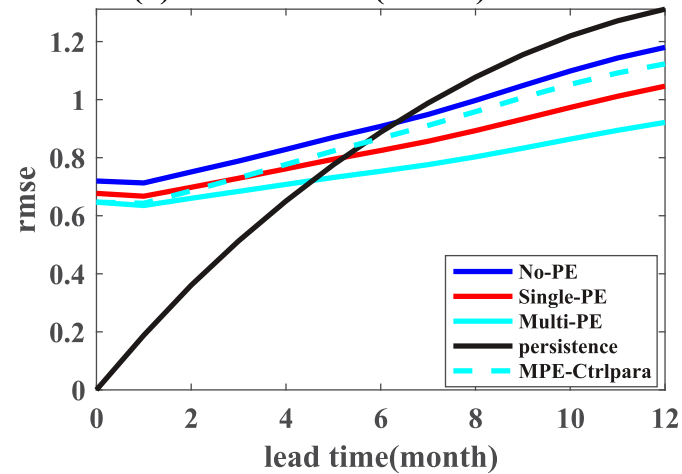

FIG. 10. As in Fig. 9, but only for the ensemble means. Additionally, we also plot two sensitivity experiments: (a), (c) MPE-Ctrlini (cyan dash), in which the forecast is made using the optimized parameters, but the No-PE initial field; (b),(d) MPE-Ctrlpara (cyan dash), in which the forecast is made using the default parameters, but the initial field from the Multi-PE.

multiple parameter estimation can also improve the forecast skills in this case. Figure 11 compares the forecast skills using the default parameter (blue) with those using the optimized parameter gam 2 (red) and the optimized multiple parameters (cyan), all with the bias correction in each forecast model. Similar to the forecast without bias correction in section 3, after single (multiple) parameter estimation, the forecast skill is increased over the default model in both ACC and RMSE. In addition, there is a further improvement of multiparameter case over the single-parameter case. In the other type of experiments, we regard the bias-correction module as a component of the model, that is, we perform the parameter estimation with the state ensembles after the bias correction and evaluate the optimized parameters by the forecast also with the bias-correction module. The results are very similar to the former case (not shown). Therefore, the improved forecast skills after parameter optimization seem to be robust regardless of the bias correction.

\section{b. Geographic-dependent parameter optimization}

Although the optimized parameters improve the forecast skill over most of the eastern and central Pacific, the forecast skill is not increased clearly over the region of the western Pacific warm pool. This can be seen in Fig. 12 in the forecast skill of the Niño-4 SST anomaly (Fig. 12). (This can also been seen more clearly in the spatial pattern of the forecast skill in the tropical Pacific at lead time of 6 months later in Figs. 15 a,b). It is seen that after multiparameter optimization, the Niño-4 forecast skill is lower than the default model in ACC and RMSE, with a lower ACC and higher RMSE. The possible cause of this poor forecast in the warm pool region is that the warm pool SSTa variability is much weaker than that in the central and eastern Pacific, and the upwelling mechanism controlled by the parameters is also much stronger in the eastern Pacific. This will lead to larger sensitivities in the central and eastern Pacific; as such, the parameter optimization is likely effective mainly on the SSTa in this region. This is confirmed in the spatial map of the parameter sensitivities in Fig. 13. The sensitivities of all parameters are large in the eastern Pacific and small in the Niño-4 region. Therefore, if the parameters are basin averaged during estimation, the optimized parameters will not be appropriate for the Niño-4 region.

As a strategy to account for the spatially varying parameter sensitivity across different regions, we performed 
(a).leadtime-acc(nino34) 1982-2000

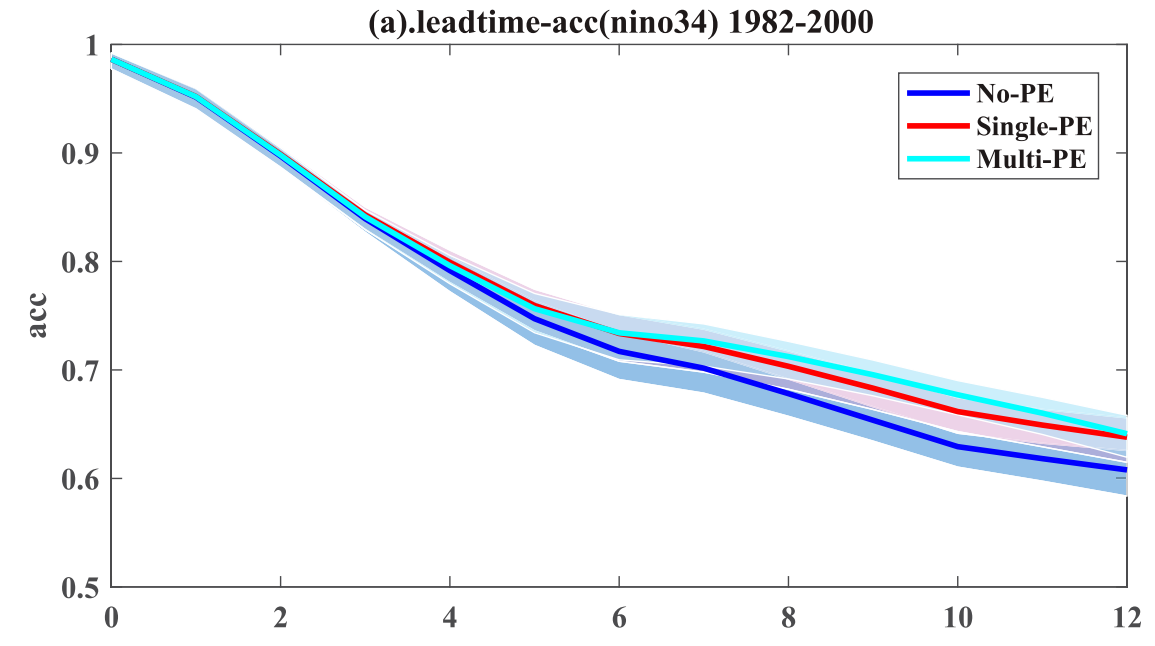

(b).leadtime-rmse(nino34) 1982-2000

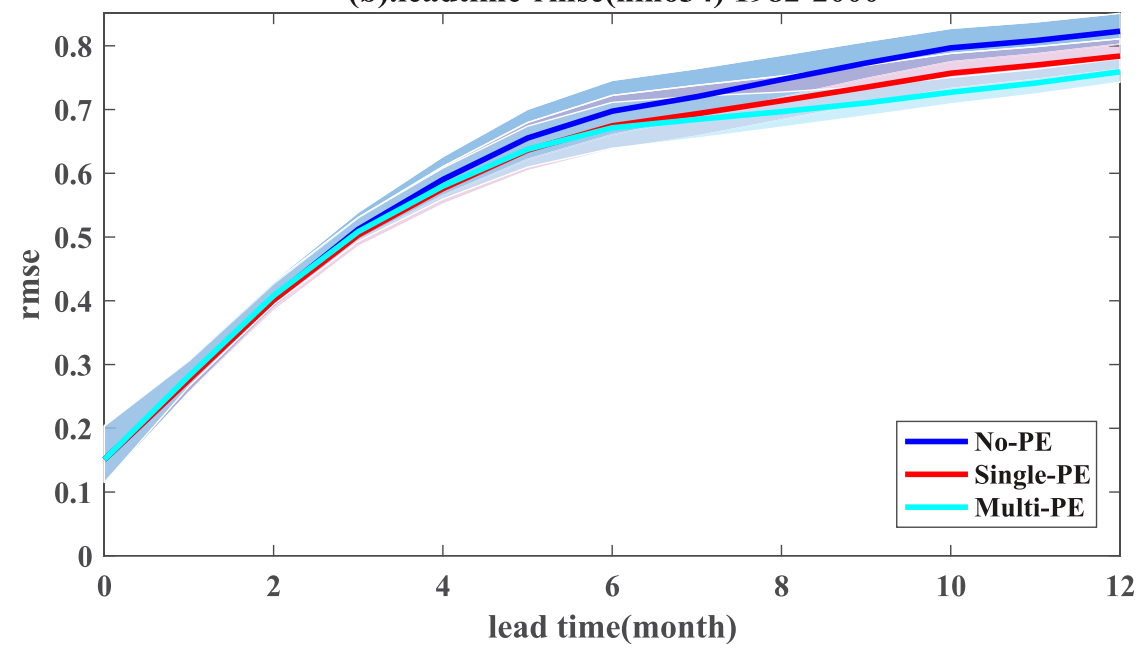

FIG. 11. Prediction skill in the LDOE5 model (ZC model with a bias correction module) of the Niño-3.4 index in the period of 1982-2000 with different lead time in the multiple parameter estimation case. The blue line is the skill of model prediction with the default parameter values. The red line is the skill of the model prediction after the singleparameter estimation of gam2. The cyan line is the skill of model prediction after multiple parameter estimation. The shading is the one standard deviation for spread. (a) ACC and (b) RMSE.

geographic-dependent parameter optimization (GPO), in which the optimized parameter values are estimated different in different regions (Wu et al. 2012). The parameters are now allowed to vary zonally, and the zonal variation of the estimated parameter is derived as the average of the last 10 years ensemble mean at each longitude, as shown in Fig. 14 (blue lines). The optimized parameters show a significant difference in the western Pacific from the eastern Pacific. The spatially varying parameters lead to a much enhanced prediction skill in the western Pacific over Multi-PE case (yellow in Fig. 12), while maintaining the forecast skill increase in the eastern and central Pacific comparable with that in the model with the spatially uniform parameter estimation discussed in section 4a. This can be seen more clearly in comparison of the SSTa forecast skills improvement over No-PE in the equatorial Pacific at lead-time 6 months in Fig. 15. The forecast skill in the uniform parameter case of Multi-PE is decreased in the western Pacific, opposite to the rest of the ocean (Figs. 15a,b). However, with GPO this decrease of forecast skill in the western Pacific is reduced in in ACC, and even diminishes in RMSE (Figs. 15c,d), while the forecast skill in the Eastern Pacific still increases. The improvement of the GPO over Multi-PE is mainly in the western Pacific (Figs. 15e,f). Therefore, 


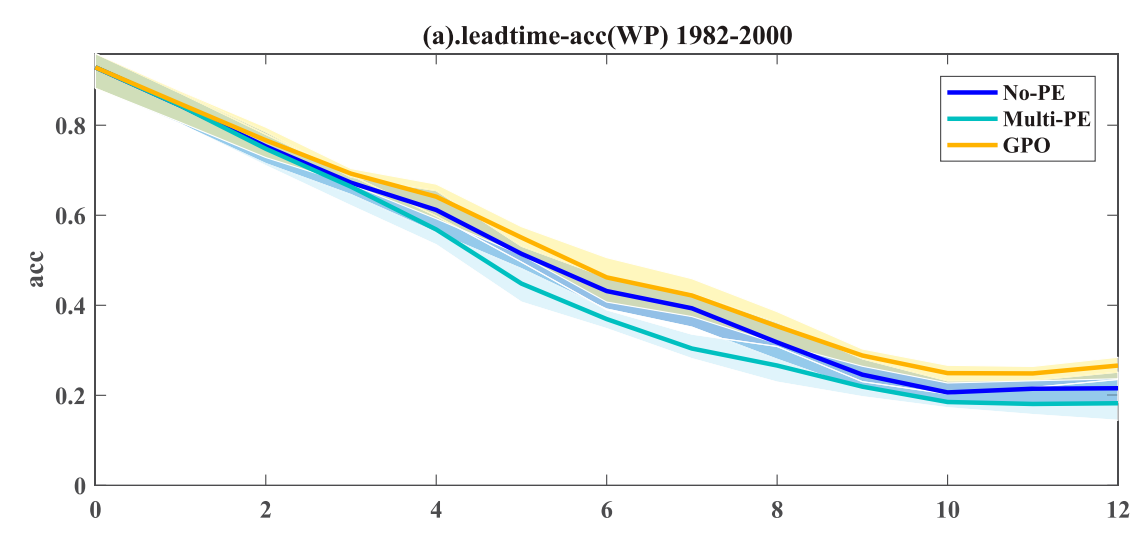

(b).leadtime-rmse(WP) 1982-2000

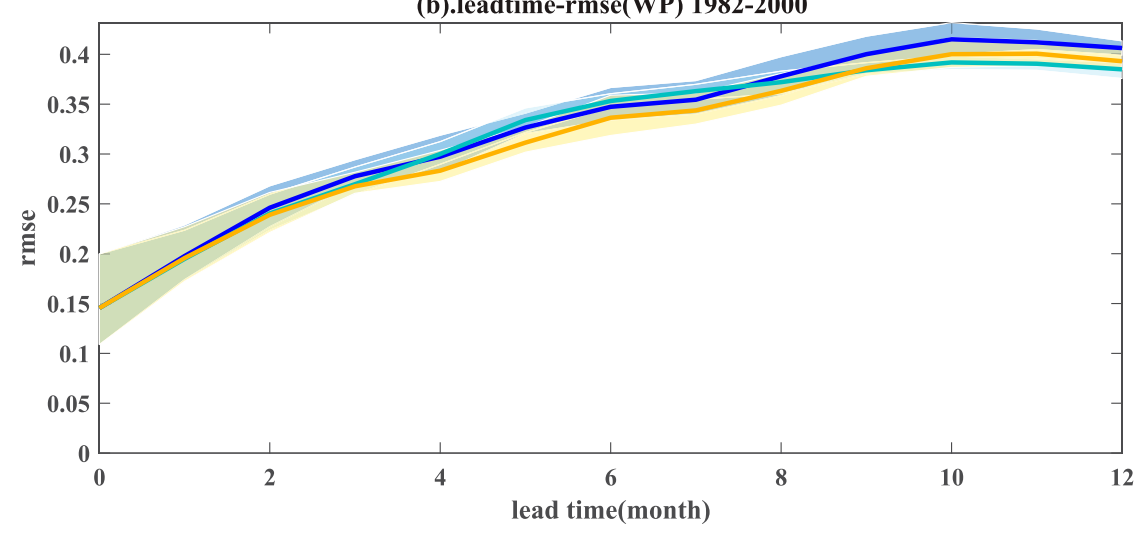

FIG. 12. Model prediction skill of Niño-4 index in 1982-2000 with different lead time in GPO case. The blue line is the skill of model simulation with the default parameter values. The cyan line is the skill of model prediction after multiple parameter estimation. The yellow line is the skill of model prediction after GPO. The shading is the one standard deviation of spread. (a) ACC and (b) RMSE.

the geographic-dependent parameter optimization can improve the forecast in the whole basin. The spatial variation of the estimated parameters may not be completely a mathematical overfitting. Indeed, there is no reason that these parameters have to be exactly spatially uniform. Therefore, the geographically dependent parameter optimization may provide a strategy to estimate the spatial structure of these parameters.

\section{Summary}

We performed parameter estimation on the ZC model for the real-world scenario using the data assimilation method of EnKF. Model parameters are estimated with model state variables simultaneously, using observational data, and the effect of parameter optimization is evaluated in terms of the ensemble forecast skill of real-world ENSO. Both single-parameter and multiparameter experiments are performed. In both cases, the parameters converge to new parameter values. The optimized parameters improve the real word ENSO prediction significantly, regardless of the bias-correction process. The largest improvement is contributed by the parameter of the largest climate sensitivity. The forecast skill is improved by the improvements in both the initial field and the model itself. A generalization to allow for the spatial variation of the estimated parameter further improves the forecast skill over the western Pacific SSTa as well as in the central and eastern Pacific.

To our knowledge, this work is the first parameter estimation work in a coupled climate model for the real-world scenario. Given the challenges in the improvement of coupled climate models in general, our work suggests that parameter estimation using data assimilation may provide an effective approach to improve climate models and climate prediction in the future. Much further study however is needed in the future for the optimization of parameters in coupled climate models. For example, EnKF is an appropriate 
(a).spatial sensitivity of gam1

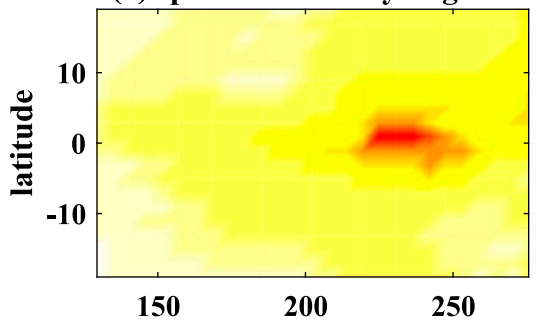

(c).spatial sensitivity of tda1

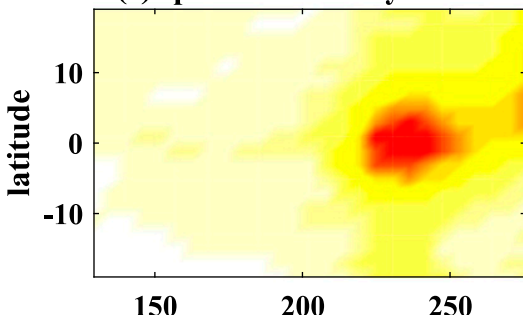

(e).spatial sensitivity of tdb1

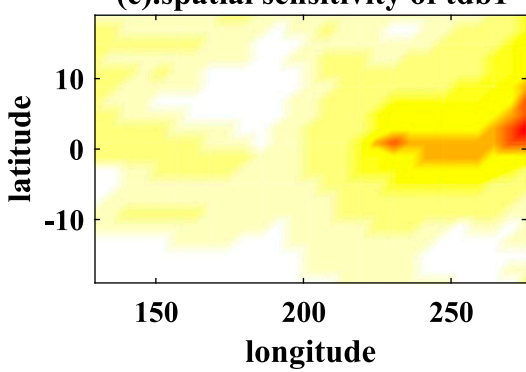

(b).spatial sensitivity of gam2

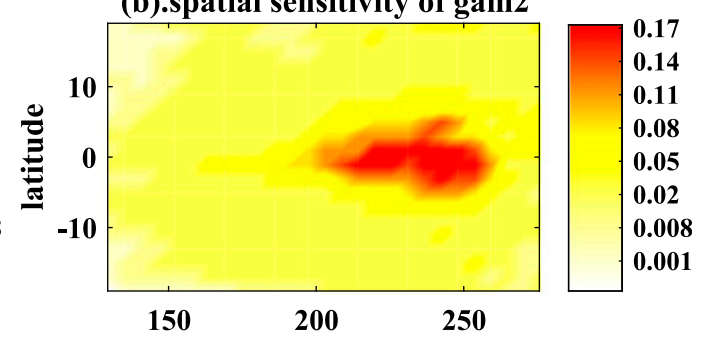

(d).spatial sensitivity of tda2

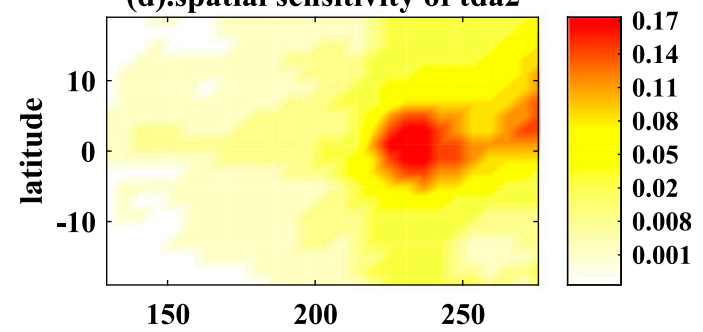

(f).spatial sensitivity of $\mathrm{tdb2}$

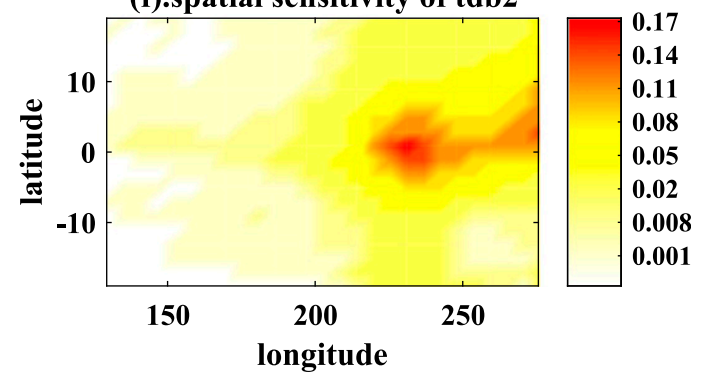

FIG. 13. One-year mean spatial sensitivity distribution of six parameters: (a) gam1, (b) gam2, (c) tda1, (d) tda2, (e) tdb1, and (f) tdb2.

method only for the case of Gaussian hypothesis and linear observation operator (such as in our study). Therefore, other methods such as particle filtering (PF) can also be performed as a "gold standard" of the results, and the GIGG-EnKF method (Bishop 2016) can also be tested when the observational error is nonnormal. Furthermore, the choice of the 6 parameters selected here is somewhat ad hoc. There are more parameters in the model. A future question is therefore how to select the optimal set of parameters for optimization. This will be studied in the future.

Acknowledgments. We thank Dr. Wansuo Dun for discussions and sharing of a ZC model in the early stage of the work. We also thank Dr. Dake Chen for sharing the LDEO5 model. This work is supported by
Chinese MOST 2017YFA0603801, NSFC41630527, and U.S. NSF AGS1656907.

\section{APPENDIX A}

\section{The SST Equation of the Zebiak-Cane Model and Parameters}

The SST equation and parameters of the model are shown below:

$$
\begin{aligned}
\frac{\partial T}{\partial t}= & -\mathbf{u}_{1} \cdot \nabla(\bar{T}+T)-\overline{\mathbf{u}_{1}} \cdot \nabla T \\
& -[\operatorname{gam} 1 \times \operatorname{HF}(\bar{w})+\operatorname{gam} 2 \times \operatorname{GF}(\bar{w}+w)] \\
& \times \frac{T-T e}{H}-\operatorname{gam} 2 \times \operatorname{GF}(\bar{w}+w) \times \overline{T_{z}}-\alpha T,
\end{aligned}
$$

$$
T_{e}=\left\{\begin{array}{ll}
\operatorname{tda} 1 \times\{\tanh [\operatorname{tdb} 1 \times(\bar{h}+\mathrm{h})]-\tanh (\operatorname{tdb} 1 \times \bar{h})\}, & h>0 \\
\operatorname{tda} 2 \times\{\tanh [\operatorname{tdb} 2 \times(\bar{h}-\mathrm{h})]-\tanh (\operatorname{tdb} 2 \times \bar{h})\}, & h<0
\end{array} .\right.
$$



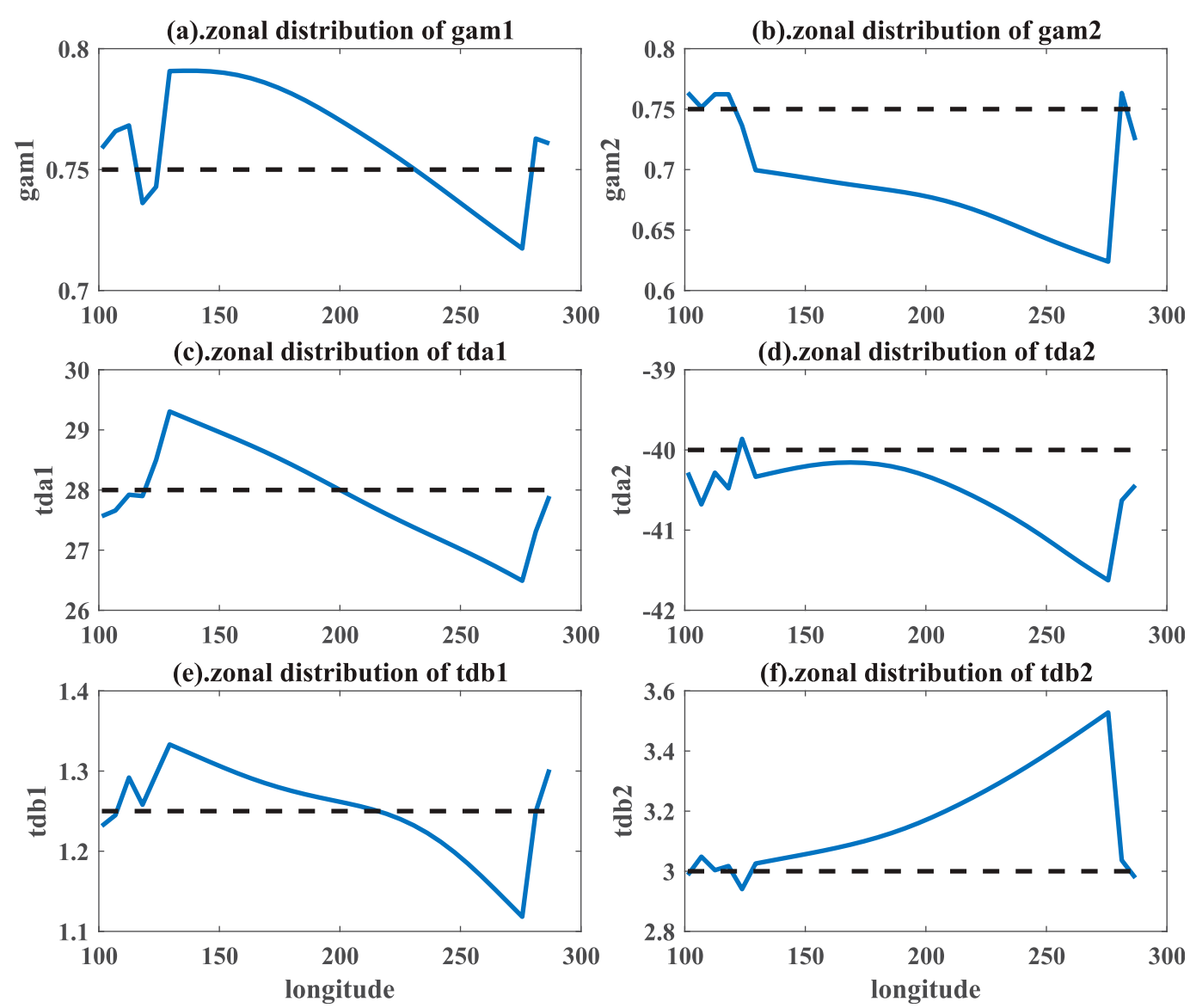

FIG. 14. GPO result of six parameters: (a) gam1, (b) gam2, (c) tda1, (d) tda2, (e) tdb1, and (f) tdb2. The horizontal axis is longitude. The ensemble size is 100 . The value (blue line) is the average of the last 10 years ensemble mean. The dashed line is the default value.

Here, $\overline{T_{z}}$ is the prescribed mean vertical temperature gradient, $T_{e}$ is the entrainment temperature, and $\bar{h}$ is the prescribed mean upper-layer depth.

Here,

$$
\begin{gathered}
\operatorname{HF}(\bar{w})\left\{\begin{array}{cc}
\bar{w}, & \bar{w}>0 \\
0 & \bar{w}<0
\end{array}\right. \\
\operatorname{GF}(\bar{w}+w)=\left\{\begin{array}{cc}
0, & \bar{w}+w<0, \bar{w}<0 \\
\bar{w}+w, & \bar{w}+w>0, \bar{w}<0 \\
-\bar{w}, & \bar{w}+w<0, \bar{w}>0 \\
w, & \bar{w}+w>0, \bar{w}>0
\end{array}\right.
\end{gathered}
$$

\section{APPENDIX B}

\section{Data Assimilation and Parameter Estimation Schemes}

\section{a. Data assimilation (DA) scheme}

On every assimilation step (the last time step of each month), read in the observational data (SSTa, UV wind stress), which are interpolated to the model grid. Obtain the observation ensemble by adding Gaussian random numbers with zero mean and standard deviation of $0.1 \mathrm{~K}$ and $0.1 \mathrm{~m} \mathrm{~s}^{-1}$ to the observation data. Perform state inflation (Hamill and Whitaker 2005), and on each observational grid, update the model variables by Eq. (B1). Integrate the model until next assimilation step.

\section{b. Parameter estimation scheme}

Step 1: Perturb the parameters by adding Gaussian random numbers (ensemble number is 100) to the default parameter values. The Gaussian random numbers have the mean of zero and the standard deviation as $20 \%$ of the default parameter values.

Step 2: Perturb the initial model SST anomaly by Gaussian random numbers with mean of zero and standard deviation of $0.1 \mathrm{~K}$ and integrate the model with the ensemble parameters for 1 year, until the ensemble spread of variables to be stable.

Step 3: Perform data assimilation described in Eq. (B1) until the model reach the quasi-equilibrium state, which is defined as the model state constrained 

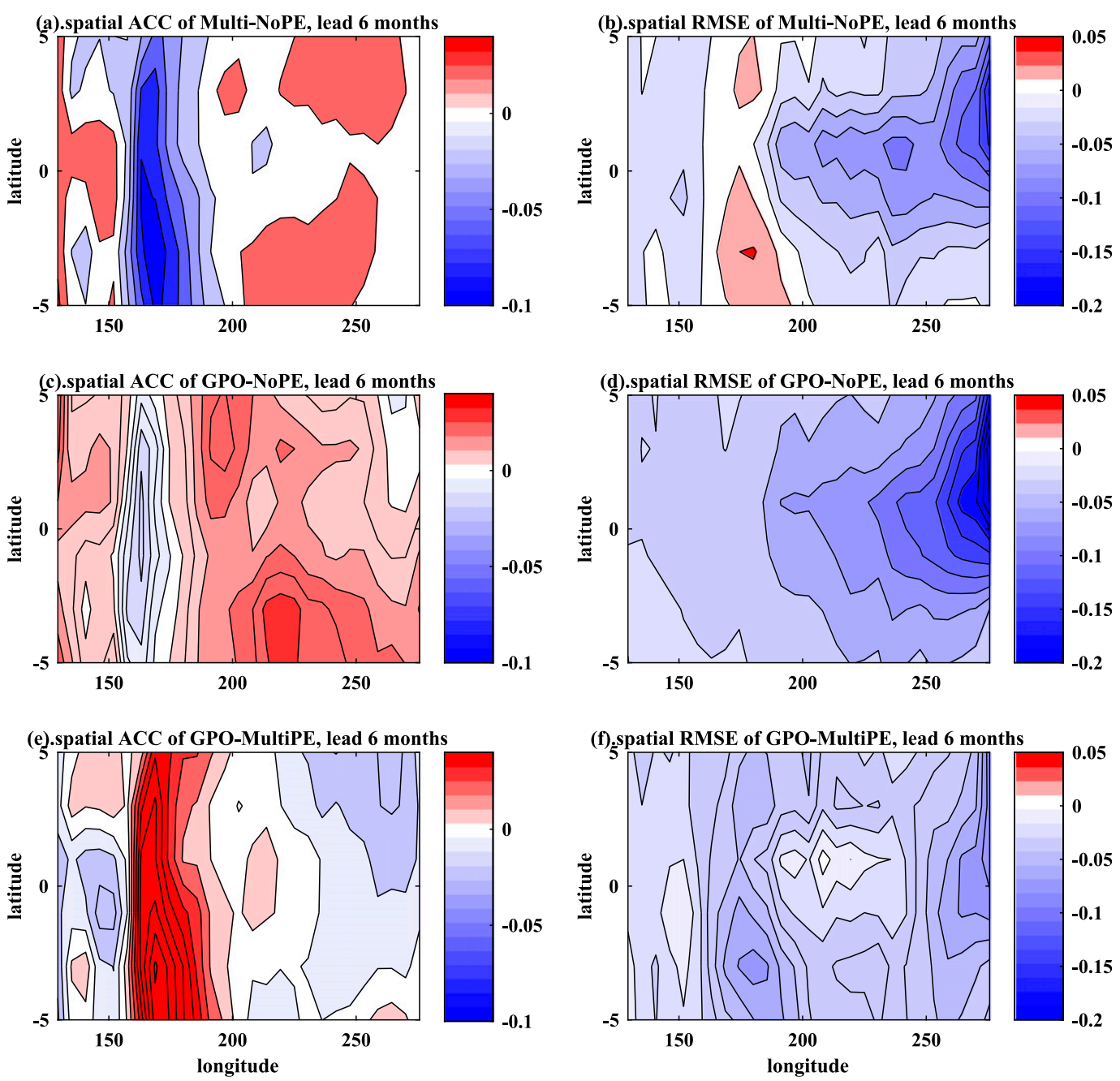

FIG. 15. The comparison of forecast skills at lead 6 months. (a) ACC and (b) RMSE of Multi-PE minus No-PE.

(c) ACC and (d) RMSE of GPO minus No-PE. (e) ACC and (f) RMSE of GPO minus Multi-PE.

enough by the observations and the model errors become stable. We choose 2 years for this period. After this period, on every assimilation step, perform data assimilation and then perform parameter estimation by Eq. (B4). After all the observations are applied to all the estimated parameters, average the parameters of the whole basin for each ensemble member. Check the spread of the parameter ensembles and do inflation by Eq. (B3).

Step 4: Integrate the model with the updated parameter ensembles until next assimilation step.

\section{1) State Assimilation}

$$
\Delta x_{n, i}=w \times \frac{\operatorname{cov}\left(x_{n}^{b}, y_{k}^{b}\right)}{\sigma_{y b}^{k 2}+\sigma_{y o}^{k 2}} \Delta y_{k, i}^{o}
$$

$$
\begin{gathered}
\Delta y_{k, i}^{o}=y_{k, i}^{o}-y_{k, i}^{b}, \\
x_{n, i}^{a}=x_{n, i}^{b}+\Delta x_{n, i} .
\end{gathered}
$$

Here $n$ represents the $n$th assimilation grid and $k$ represents the $k$ th observational grid, which goes by $k=1$, $2, \ldots$ from the first observational grid to the end. Parameter $\Delta y_{k, i}^{o}$ is the innovation on $k$ th observational grid of $i$ th ensemble member, which is the difference between the observation and background of variable $y$. Parameters $x_{n}^{b}, y_{k}^{b}$ are the model state ensemble of $x$ on assimilation grid and model-estimated ensemble of variable $y$ on observational grid. Parameters $\sigma_{y b}^{k 2}$ and $\sigma_{y o}^{k 2}$ are the variance of model-estimated ensemble of $y$ and observation ensemble on observational grid. Parameters $x_{n, i}^{a}$ and $x_{n, i}^{b}$ are the analysis and background of model state on assimilation grid. Parameter $w$ is the covariance 
localization factor obtained by the fifth-order function (Gaspari and Cohn 1999):

$$
w=\left\{\begin{array}{cl}
-\frac{z^{5}}{4}+\frac{z^{4}}{2}+\frac{5 z^{3}}{8}-\frac{5 z^{2}}{3}+1, & 0 \leq z \leq 1 \\
\frac{z^{5}}{12}-\frac{z^{4}}{2}+\frac{5 z^{3}}{8}+\frac{5 z^{2}}{3}-5 z+4-\frac{2}{3 z}, & 1 \leq z \leq 2 \\
0 & z \geq 2
\end{array} .\right.
$$

Here, $z=D / D_{0}, D$ is the distance between the observational grid and the assimilation grid, and $D_{0}$ is the prescribed "influence radius," which is $1500 \mathrm{~km}$ in our study.

\section{2) INFLATION METHOD}

An additive inflation technique will be applied to the state:

$$
\begin{aligned}
x_{i} & =x_{i}+\eta_{i} \\
\eta_{i} & =s[x(\operatorname{tr})-\bar{x}] .
\end{aligned}
$$

Here tr is a randomly time from the time series of the model free run, $\bar{x}$ is the mean climatological state, and $s$ is a scaling factor which equals to 0.25 here.

We inflate the parameters by a conditional covariance inflation. That is, when the parameter ensemble spread is below a threshold value, we inflate the spread back to this value:

$$
\beta_{i}=\bar{\beta}+\frac{a}{\sigma_{\beta}} \times\left(\beta_{i}-\bar{\beta}\right)
$$

Here $\beta_{i}$ is the $i$ th member of parameter ensemble, $\bar{\beta}$ is the ensemble mean, $a$ is the threshold value, and $\sigma_{\beta}$ is the standard deviation of the parameter ensemble.

\section{3) Parameter estimation}

$$
\begin{aligned}
\Delta \beta_{n, i} & =w \times \frac{\operatorname{cov}\left(\beta_{n}^{b}, y_{k}^{b}\right)}{\sigma_{y b}^{k 2}+\sigma_{y o}^{k 2}} \Delta y_{k, i}^{o} \\
\beta_{n, i}^{a} & =\beta_{n, i}^{b}+\Delta \beta_{n, i} .
\end{aligned}
$$

Here $n$ represents the $n$th assimilation grid and $k$ represents the $k$ th observational grid, which goes by $k=1,2, \ldots$ from the first observational grid to the end. Parameter $\Delta y_{k, i}^{o}$ is the innovation on $k$ th observational grid of $i$ th ensemble member. Parameters $\beta_{n}^{b}, y_{k}^{b}$ are the parameter ensemble on assimilation grid and modelestimated ensemble of variable $y$ on observational grid. Parameters $\sigma_{y b}^{k 2}$ and $\sigma_{y o}^{k 2}$ are the variance of modelestimated ensemble of $y$ and observation ensemble on observational grid.

\section{REFERENCES}

Aksoy, A., F. Zhang, and J. W. Nielsen-Gammon, 2006a: Ensemble-based simultaneous state and parameter estimation with MM5. Geophys. Res. Lett., 33, L12801, https://doi.org/10.1029/ 2006 GL026186.

$\longrightarrow, \ldots$, and - 2006b: Ensemble-based simultaneous state and parameter estimation in a two-dimensional sea-breeze model. Mon. Wea. Rev., 134, 2951-2970, https://doi.org/10.1175/ MWR3224.1.

Anderson, J. L., 2001: An ensemble adjustment Kalman filter for data assimilation. Mon. Wea. Rev., 129, 2884-2903, https://doi.org/ 10.1175/1520-0493(2001)129<2884:AEAKFF>2.0.CO;2.

Bishop, C. H., 2016: The GIGG-EnKF: Ensemble Kalman filtering for highly skewed non-negative uncertainty distributions. Quart. J. Roy. Meteor. Soc., 142, 1395-1412, https://doi.org/10.1002/qj.2742.

Chen, D., S. E. Zebiak, R. Canizares, and A. Kaplan, 2000: Bias correction of an ocean-atmosphere coupled model. Geophys. Res. Lett., 27, 2585-2588, https://doi.org/10.1029/1999GL011078.

_ A. Kaplan, S. E. Zebiak, and D. Huang, 2004: Predictability of El Niño over the past 148 years. Nature, 428, 733-736, https://doi.org/10.1038/nature02439.

Evensen, G., 1994: Sequential data assimilation with a nonlinear quasi-geostrophic model using Monte Carlo methods to forecast error statistics. J. Geophys. Res., 99, 10143-10162, https://doi.org/10.1029/94JC00572.

Gaspari, G., and S. E. Cohn, 1999: Construction of correlation functions in two and three dimensions. Quart. J. Roy. Meteor. Soc., 125, 723-757, https://doi.org/10.1002/qj.49712555417.

Goldenberg, S. B., and J. J. O’Brien, 1981: Time and space variability of tropical Pacific wind stress. Mon. Wea. Rev., 109, 1190-1207, https://doi.org/10.1175/1520-0493(1981)109<1190: TASVOT $>2.0 . \mathrm{CO} ; 2$.

Hamill, T. M., and J. S. Whitaker, 2005: Accounting for the error due to unresolved scales in ensemble data assimilation: A comparison of different approaches. Mon. Wea. Rev., 133, 3132-3147, https://doi.org/10.1175/MWR3020.1.

Hansen, J., and C. Penland, 2007: On stochastic parameter estimation using data assimilation. Physica $D, 230,88-98$, https:/ doi.org/10.1016/j.physd.2006.11.006.

Hu, X.-M., F. Zhang, and J. W. Nielsen-Gammon, 2010: Ensemblebased simultaneous state and parameter estimation for treatment of mesoscale model error: A real-data study. Geophys. Res. Lett., 37, L08802, https://doi.org/10.1029/2010GL043017.

Karspeck, A. R., and J. L. Anderson, 2007: Experimental implementation of an ensemble adjustment filter for an intermediate ENSO model. J. Climate, 20, 4638-4658, https:// doi.org/10.1175/JCLI4245.1.

Kondrashov, D., C.-J. Sun, and M. Ghil, 2008: Data assimilation for a coupled ocean-atmosphere model. Part II: Parameter estimation. Mon. Wea. Rev., 136, 5062-5076, https://doi.org/ 10.1175/2008MWR2544.1.

Li, S., and Coauthors, 2018: Estimating convective parameters in the GFDL CM2.1 model using ensemble data assimilation. J. Adv. Model. Earth Syst., 10, 989-1010, https://doi.org/ 10.1002/2017MS001222.

Liu, Y., Z. Liu, S. Zhang, X. Rong, R. Jacob, S. Wu, and F. Lu, 2014: Ensemble based parameter estimation in a coupled GCM using the adaptive spatial average method. J. Climate, 27, 4002-4014, https://doi.org/10.1175/JCLI-D-13-00091.1.

Liu, Z., Y. Jin, and X. Rong, 2019: A theory for the seasonal predictability barrier: threshold, timing, and intensity. J. Climate, 32, 423-443, https://doi.org/10.1175/JCLI-D-18-0383.1. 
Lu, F., Z. Liu, Y. Liu, S. Zhang, and R. Jacob, 2017: Understanding the control of extratropical atmospheric variability on ENSO using a coupled data assimilation approach. Climate Dyn., $\mathbf{4 8}$, 3139-3160, https://doi.org/10.1007/s00382-016-3256-7.

Menemenlis, D., I. Fukumori, and T. Lee, 2005: Using Green's functions to calibrate an ocean general circulation model. Mon. Wea. Rev., 133, 1224-1240, https://doi.org/10.1175/ MWR2912.1.

Tong, M., and M. Xue, 2008a: Simultaneous estimation of microphysical parameters and atmospheric state with simulated radar data and ensemble square root Kalman filter. Part I: Sensitivity analysis and parameter identifiability. Mon. Wea. Rev., 136, 1630-1648, https://doi.org/10.1175/2007MWR2070.1. , and - 2008b: Simultaneous estimation of microphysical parameters and atmospheric state with simulated radar data and ensemble square root Kalman filter. Part II: Parameter estimation experiments. Mon. Wea. Rev., 136, 1649-1668, https://doi.org/10.1175/2007MWR2071.1.

Torrence, C., and P. J. Webster, 1998: The annual cycle of persistence in the El Niño/Southern Oscillation. Quart. J. Roy. Meteor. Soc., 124, 1985-2004, https://doi.org/10.1002/qj.49712455010.

Toyoda, T., and Coauthors, 2015: An improved simulation of the deep Pacific Ocean using optimally estimated vertical diffusivity based on the Green's function method. Geophys. Res. Lett., 42, 9916-9924, https://doi.org/10.1002/ 2015 GL065940.
Wu, X., S. Zhang, Z. Liu, A. Rosati, T. Delworth, and Y. Liu, 2012: Impact of geographic-dependent parameter optimization on climate estimation and prediction: simulation with an intermediate coupled model. Mon. Wea. Rev., 140, 3956-3971, https://doi.org/10.1175/MWR-D-11-00298.1.

Zebiak, S. E., and M. A. Cane, 1987: A model El Nino-Southern Oscillation. Mon. Wea. Rev., 115, 2262-2278, https://doi.org/ 10.1175/1520-0493(1987)115<2262:AMENO>2.0.CO;2.

Zhang, S., Z. Liu, A. Rosati, and T. Delworth, 2012: A study of enhancive parameter correction with coupled data assimilation for climate estimation and prediction using a simple coupled model. Tellus, 64A, 10963 , https://doi.org/10.3402/ tellusa.v64i0.10963.

Zheng, F., and J. Zhu, 2016: Improved ensemble-mean forecasting of ENSO events by a zero-mean stochastic error model of an intermediate coupled model. Climate Dyn., 47, 3901-3915, https://doi.org/10.1007/s00382-016-3048-0.

,-- , R.-H. Zhang, and G.-Q. Zhou, 2006: Ensemble hindcasts of SST anomalies in the tropical Pacific using an intermediate coupled model. Geophys. Res. Lett., 33, L19604, https://doi.org/10.1029/2006GL026994.

Zhu, Y., and I. M. Navon, 1999: Impact of parameter estimation on the performance of the FSU Global Spectral Model using its full physics adjoint. Mon. Wea. Rev., 127, 1497-1517, https://doi.org/10.1175/1520-0493(1999)127<1497: IOPEOT $>2.0 . \mathrm{CO} ; 2$. 